\title{
Factores asociados a la decisión paterna sobre el retorno a clases presenciales en Colombia durante la pandemia COVID-19
}

\section{Associated factors with the paternal decision on the return to face-to-face classes in Colombia during the COVID-19 pandemic}

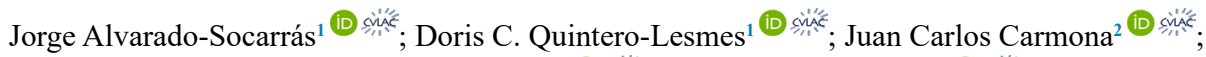

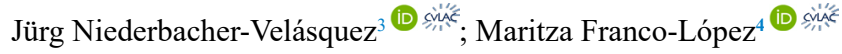

Forma de citar: Alvarado Socarras J, Quintero Lesmes D, Carmona JC, Niederbacher Velásquez J, Franco López M. Factores asociados a la decisión paterna sobre el retorno a clases presenciales en Colombia durante la pandemia COVID-19. Salud UIS. 53: e21018. doi: https://doi.org/10.18273/saluduis.53.e:21018 (c) (i)

\section{Resumen}

Introducción: la pandemia por SARS-CoV-2 generó el cierre de los colegios y el inicio de la educación virtual, como medida para disminuir la propagación del virus. Rápidamente, se planteó el regreso a los colegios; sin embargo, es probable que exista un rechazo de los padres por el regreso a clases de forma presencial o mediante alternancia. Por esta razón, se evaluaron los factores asociados a esta oposición. Material y métodos: se realizó un estudio analítico de corte transversal mediante una encuesta, dirigida a padres que acuden a consultorios con sus hijos en varias ciudades de Colombia. Se determinó el porcentaje de padres que deseaban mantener la escolarización mediante la virtualidad y los factores asociados que motivan a no aceptar el pronto retorno a clases presenciales. Se realizó un análisis de regresión binomial para estimar razones de prevalencia (RP), ajustado a la edad de los padres, tomando como variable dependiente la aceptación de la virtualidad como estrategia para no regresar a los colegios, y se determinaron los factores de mayor importancia en la no aceptación del retorno a clases. Resultados: el 81,06\% de los padres no acepta el retorno a clase de forma presencial. El principal factor asociados a esta decisión fue que alguno de los padres presentara alguna comorbilidad, principalmente, hipertensión y obesidad. Tener hijos mayores de 10 años (RP: 2,64: IC 95\%: 1,48-4,72), convivir con personas mayores de 70 años (RP: 2,2; IC 95\%; 1,46-3,29) y conocer alguna persona fallecida por COVID-19 (RP: 1,86; IC 95\%: 1,42-2,44) también fueron asociadas a no aceptar el retorno a clase presencial. Conclusiones: existe una clara tendencia a mantener la virtualidad como opción educativa en Colombia para el no regreso a clases presenciales en población de estrato medio y alto. Los factores asociados sugieren una forma de protección familiar. Esta evidencia debe considerarse específica para el periodo de recolección de datos y el grupo incluido en el estudio. Se requieren estudios en los estratos socioeconómicos más bajos, donde se supone ocurren más efectos adversos asociados al no retorno a clases presenciales.

Palabras clave: COVID-19; SARS-CoV-2; Pandemia; Escuelas; Niños; Adolescentes.

1. Fundación Cardiovascular de Colombia. Floridablanca, Colombia.

2. Hospital Infantil Napoleón Franco Pareja. Cartagena, Colombia.

3. Universidad Industrial de Santander. Bucaramanga, Colombia.

4. Compensar. Consultorio Particular. Bogotá, Colombia.

Correspondencia: Jorge Alvarado Socarras. Dirección: FCC Calle 155A No. 23 - 58 Urbanización El Bosque. Teléfono: (+57) 3103411716. Correo electrónico: jorgealvarado@fcv.org 


\begin{abstract}
Introduction: The SARS-CoV-2 pandemic led to the closure of schools and the beginning of virtual education, as a measure to reduce the spread of the virus. Shortly after, there was a planned return to in-person learning, however, there existed the possibility of parents rejecting the notion of returning to in-person learning or a hybrid model of inperson and online learning, there is likely a rejection of parents to return to classes in person. For this reason, the factors possibly associated with this opposition were evaluated. Material and methods: A cross-sectional analytical study was carried out through an online survey aimed at parents who attend private clinics with their children, in several cities of Colombia. The percentage of parents who wished to maintain their children's schooling through virtuality and the factors associated with not accepting the prompt return to face-to-face classes were determined. A binomial regression analysis, to estimate Prevalence Ratios (PR), adjusted for the age of the parents was carried out, taking as a dependent variable the acceptance of virtuality as a strategy for not returning to school and the most important factors in the nonacceptance of returning to school were determined. Results: $81.06 \%$ of the parents did not accept the return to class in person. The main factors associated with this decision were that one of the parents had some comorbidity, mainly hypertension and obesity. Having children older than 10 years (PR: 2.64; 95\% CI: 1.48-4.72), living with people older than 70 years (PR: 2.2; 95\% CI;1.46-3.29), and knowing someone who died from COVID-19 (PR: 1.86; 95\% CI 1.422.44) were associated with not accepting the return to face classes. Conclusions: There is a clear tendency to maintain virtuality as an educational option in Colombia for not returning to face-to-face classes in the middle and upper stratum population. Associated factors suggest a form of family protection. This evidence should be considered specific for the data collection period and the group included in the study. Studies are required in the lowest socioeconomic strata, where it is assumed that there are more adverse effects associated with not returning to face-to-face classes.
\end{abstract}

Keywords: COVID-19; SARS-CoV-2; Pandemic; Schools; Children; Adolescents.

\section{Introducción}

En diciembre del 2019, en Wuhan, provincia de Hubei, China, detectan casos de una "neumonía", de etiología desconocida $^{1}$. El 9 de enero de 2020, la Organización Mundial de la Salud (OMS) informa que las autoridades chinas determinaron que el brote se debía a un nuevo tipo de coronavirus, hoy denominado SARS-CoV-2, y que causa la enfermedad conocida como COVID-19, causante de cuadros asintomáticos, leves y neumonía severa, que puede ocasionar la muerte ${ }^{1}$, siendo motivo de gran preocupación a nivel mundial a principios del año 2020. Esta infección tuvo una rápida propagación y el 11 de marzo de 2020 la OMS la declaró pandemia ${ }^{1}$. Este virus tiene una alta transmisibilidad y una tasa de mortalidad global del 3,4\%, aproximadamente ${ }^{2}$. Por lo anterior, se inició una serie de cambios en las actividades a nivel mundial buscando disminuir la trasmisión, la saturación de los sistemas de salud y la mortalidad, entre otras. Muchas de estas estrategias tuvieron que ver con el autocuidado, como el lavado de manos, el uso de mascarillas, cuarentenas (estrictas y sectorizadas) y el distanciamiento social ${ }^{3}$.

Lo anterior, llevó a un bloqueo económico mundial, cierre de aeropuertos y disminución de actividades turísticas y educativas, entre otras ${ }^{4}$. A nivel educativo, las clases presenciales fueron clausuradas y la virtualidad fue elegida como la estrategia educativa en el momento en que se declaró la pandemia, haciendo parte del esfuerzo para minimizar la trasmisión por COVID-19. Luego de la declaración de la pandemia (marzo del 2020), aproximadamente 1,6 billones de estudiantes en 191 países del mundo suspendieron al menos de forma presencial su actividad escolar ${ }^{5}$. Esta estrategia había sido sugerida por la OMS durante la epidemia por H1N1, basados en una estrategia de mitigación de la propagación de la infección, resultado de los modelos de salud pública durante ese momento ${ }^{6}$. Lo que dio soporte a la virtualidad como opción del proceso educativo. Esta medida fue una estrategia que buscó mantener el proceso educativo y evitar retrasos en el aprendizaje y desarrollo de los niños. Sin embargo, estos cambios que generan largos periodos de aislamiento, sedentarismo, modificación en la forma de aprendizaje y socialización, podrían estar relacionados con efectos secundarios tanto físicos como psicológicos, como consecuencia de la falta de integralidad en el proceso educativo ${ }^{7}$.

Por lo anterior, el regreso a clase en la forma tradicional, o al menos métodos como la alternancia, parece ser una necesidad a corto plazo, evaluando riesgos, beneficios y condiciones propias de cada región. Además, las políticas gubernamentales están buscando reinicio de actividades presenciales, por lo menos de manera parcial. Sin embargo, existe un gran temor de muchos padres por este eventual retorno ${ }^{8}$. En Colombia, inició una cuarentena obligatoria desde el 24 de marzo del 2020, que incluyó el cierre de todas las 
escuelas y colegios a nivel nacional, que se mantiene hasta la fecha; sin embargo, a finales del 2020, se inició el proceso de alternancia en algunas regiones del país. A pesar de plantearse un retorno con alternancia de los alumnos, existe resistencia de algunos padres a esta opción. De esta manera, el objetivo de este estudio fue evaluar la posición de los padres en Colombia para un regreso a clases presenciales en el segundo semestre de 2020 y describir los factores relacionados durante la pandemia por COVID-19. Además, algunos desenlaces secundarios físicos y psicológicos también fueron evaluados.

\section{Material y métodos}

Se realizó un estudio descriptivo, analítico de corte transversal y exploratorio. Los datos fueron obtenidos a partir del diligenciamiento voluntario de una encuesta electrónica dirigida a padres y cuidadores de niños atendidos en la consulta pediátrica de médicos pediatras, de manera simultánea en las diferentes ciudades en Colombia, entre el 27 de agosto y el 14 de septiembre del año 2020. La encuesta fue creada para el estudio, previa validación de un grupo de cinco pediatras, en la plataforma de formularios de Google. Se invitó a participar a pediatras de diferentes ciudades de Colombia en la difusión de la encuesta. Esta difusión se realizó mediante el envío de un "enlace" al médico pediatra participante en el estudio, previa aceptación de la invitación, quien a su vez se encargó de remitirlo a los correos electrónicos de sus pacientes, de acuerdo con la base de datos de usuarios de su consultorio. Se incluyeron niños menores de 18 años que estuvieran matriculados en un centro educativo en Colombia y que asistieran a la consulta pediátrica del médico participante en el estudio.

Una vez diligenciada la encuesta de manera voluntaria, la información suministrada por cada uno de los participantes fue cargada en un archivo Excel, al cual solo tuvo acceso el investigador principal del estudio y el analista de datos. Se mantuvo el anonimato en todas las encuestas, dado que no se preguntaba el nombre de los participantes o algún dato que lo identificara, además el envío de la encuesta a los correos electrónicos de los pacientes lo hacía le médico pediatra tratante.

Para el análisis estadístico, inicialmente, se realizó un análisis descriptivo, en donde los valores categóricos se presentan como proporciones y las variables continuas como medias y desviación estándar (DE). Así mismo, se realizó un análisis bivariado, en donde se estableció como variable dependiente "aceptación de la virtualidad como estrategia para no enviar a sus hijos a clases presenciales" e independientes a la totalidad de variables de la encuesta. Las variables que obtuvieron una $p \geq 0,20$ en el análisis bivariado se mantuvieron para la exploración de asociación en el modelo de regresión binomial. Todos los valores de $\mathrm{p}$ se tomaron a dos colas, considerando $\mathrm{p}<0,05$ como estadísticamente significativo. La asociación entre variable dependiente (aceptación de la virtualidad como estrategia para no enviar a sus hijos a clases presenciales) e independientes (demás variables) en este estudio se evaluó utilizando modelos de regresión binomial con su correspondiente valoración de bondad de ajuste, como forma de aproximación a las razones de prevalencia (RP). Todos los datos fueron analizados en el software estadístico Stata versión 14.0 (Stata Corporation, College Station, TX).

\section{Resultados}

Un total de 1452 encuestas fueron analizadas. Se excluyeron 9 encuestas, dado que estaban duplicadas en la base de datos. El $85 \%$ de las encuestas las respondieron las madres. Ciudades capitales como Cartagena $(38,94 \%)$, Bogotá $(14,6 \%)$, Bucaramanga $(12,27 \%)$, Cali $(10,68 \%)$ y Barranquilla $(7,44 \%)$ tuvieron mayor proporción de participación. La edad promedio del padre fue de 43 y de la madre, 39. En relación con las actividades, el $89,3 \%$ de los padres laboraban presencialmente y el $43,1 \%$ lo hacía de forma virtual. Con relación a las áreas de trabajo, el $22,1 \%$ trabajaban en: administración-gerencia; $18,9 \%$, en salud; $17,01 \%$, en ingeniería; $7,8 \%$, en derechodocencia; $7,09 \%$, independientes; y $27 \%$, en otras actividades. Por su parte, el $73,9 \%$ de las madres laboraban; 46,1\%, de forma virtual. En las áreas de trabajo: $35,5 \%$ trabajaba en otras actividades; $26 \%$ en áreas de la salud; $16,1 \%$ en administración-gerencia; 9,4\% en derecho-docencia; $6,6 \%$ en ingenierías; y $6,2 \%$ independientes. El $87,2 \%$ tenía entre 1 y 2 hijos. El $56,5 \%$ de los padres no presentan ninguna morbilidad. El $56,9 \%$ de los padres conviven con personas sin comorbilidad. Otras variables que se consideraron en la encuesta son descritas en la Tabla 1. Se encontró diferencia entre los padres que aceptaban la virtualidad como estrategia para el no retorno a clases presenciales en variables como "si el padre o la madre presenta alguna comorbilidad, principalmente hipertensión, cardiopatía isquémica u obesidad" $(p<0,0001)$. Así mismo, convivir con personas con comorbilidades como las anotadas u otras, o adultos mayores de 70 años, tuvieron asociación en aceptar la virtualidad como estrategia educativa $(p<0,0001)$. El resto de las variables asociadas se presentan en la Tabla 2. 
Tabla 1. Descripción de las variables de la encuesta.

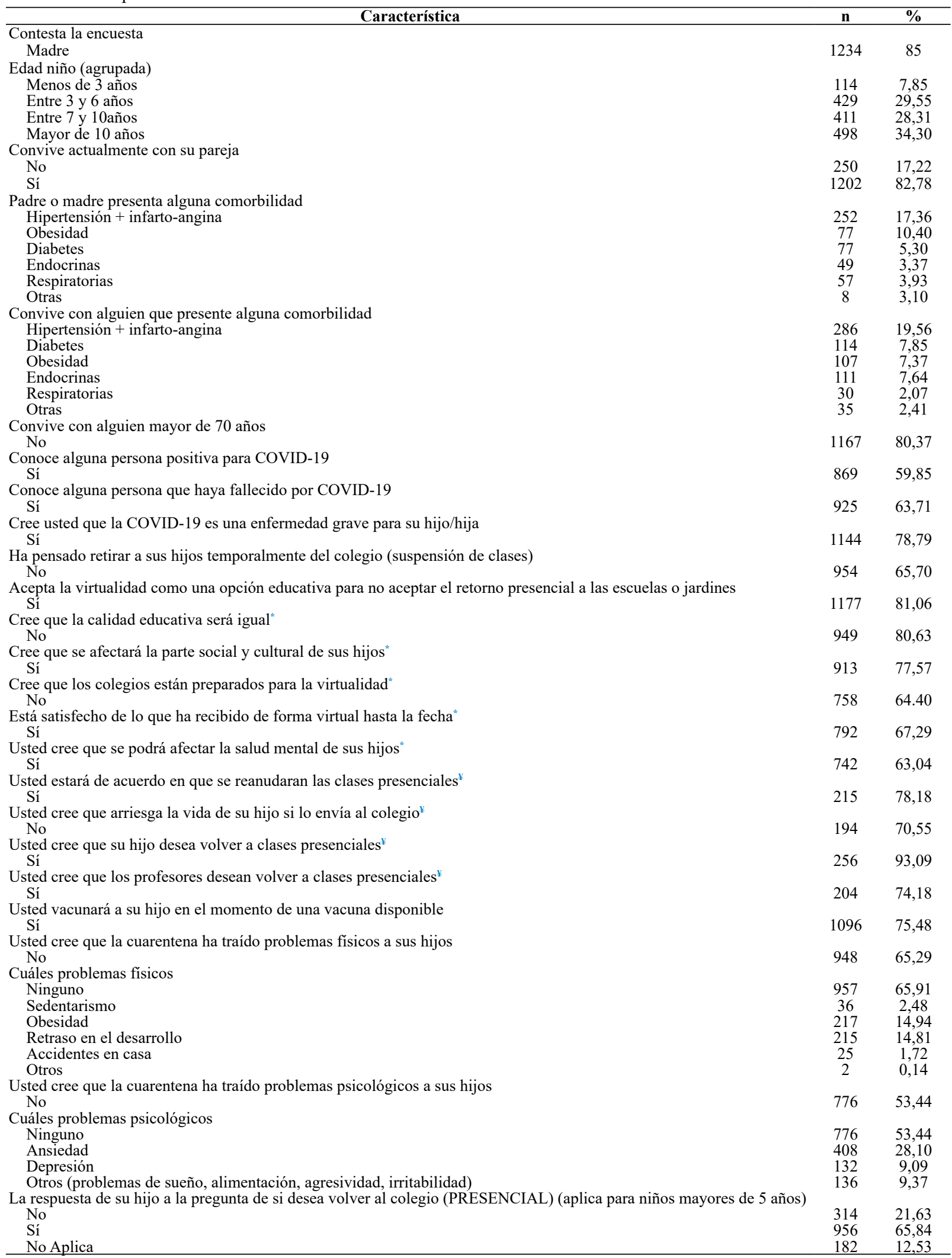

* Si a la pregunta de virtualidad contestó afirmativamente.

${ }^{\sharp} \mathrm{Si}$ a la pregunta de virtualidad contestó negativamente. 
Factores asociados a la decisión paterna sobre el retorno a clases presenciales en Colombia durante la pandemia COVID-19

Tabla 2. Prevalencias de las variables que inciden en el no retorno a clases presenciales.

\begin{tabular}{|c|c|c|c|}
\hline \multirow[b]{2}{*}{ Característica } & \multicolumn{3}{|c|}{$\begin{array}{c}\text { Acepta virtualidad para no enviar } \\
\text { a sus hijos a clases presenciales }\end{array}$} \\
\hline & $\begin{array}{l}\text { No } \\
\mathbf{n}=275\end{array}$ & $\begin{array}{c}\text { Sí } \\
\mathbf{n}=\mathbf{1 1 7 7}\end{array}$ & Valor de $\mathbf{p}^{+}$ \\
\hline \multicolumn{4}{|l|}{ Contesta la encuesta } \\
\hline Madre & $246(89,45)$ & $988(83,94)$ & $0,021^{*}$ \\
\hline Padre & $29(10,55)$ & $189(16,06$ & \\
\hline Edad del padre & $42,31(7,65)$ & $43,15(7,43)$ & $0,0044^{¥}$ \\
\hline Edad de la madre & $38,74(5,36)$ & $39,74(6,16)$ & $0,0016^{¥}$ \\
\hline \multicolumn{4}{|l|}{ Edad niño (agrupada) } \\
\hline Menos de 3 años & $26(9,45)$ & $88(7,48)$ & $<0,0001^{\dagger}$ \\
\hline Entre 3 y 6 años & $112(40,73)$ & $317(26,93)$ & \\
\hline Entre 7 y 10 años & $85(30,91)$ & $326(27,70)$ & \\
\hline Mayor de 10 años & $52(18,91)$ & $446(37,89)$ & \\
\hline \multicolumn{4}{|c|}{ Padre o madre presenta alguna comorbilidad } \\
\hline Ninguna & $209(76,00)$ & $612(52,00)$ & $<0,0001^{\dagger}$ \\
\hline Hipertensión + infarto-angina & $24(8,73)$ & $228(19,37)$ & \\
\hline Diabetes & $7(2,55)$ & $70(5,95)$ & \\
\hline Obesidad & $16(5,82)$ & $135(11,47)$ & \\
\hline Endocrinas & $7(2,55)$ & $42(3,57)$ & \\
\hline Respiratorias & $6(2,18)$ & $51(4,33)$ & \\
\hline Otras & $6(2,18)$ & $39(3,31)$ & \\
\hline \multicolumn{4}{|c|}{ Convive con alguien que presente alguna comorbilidad } \\
\hline Ninguna & $198(72)$ & $571(48,51)$ & $<0,0001^{\dagger}$ \\
\hline Hipertensión + infarto-angina & $33(12)$ & $253(21,50)$ & \\
\hline Diabetes & $14(5,09)$ & $100(8,50)$ & \\
\hline Obesidad & $9(3,27)$ & $98(8,33)$ & \\
\hline Endocrinas & $15(5,45)$ & $96(8,16)$ & \\
\hline Respiratorias & $2(0,73)$ & $28(2,38)$ & \\
\hline Otras & $4(1,45)$ & $31(2,63)$ & \\
\hline \multicolumn{4}{|c|}{ Convive con alguien mayor de 70 años } \\
\hline No & $245(80,09)$ & $922(78,33)$ & $<0,0001^{\ddagger}$ \\
\hline Sí & $30(10,91)$ & $255(21,67)$ & \\
\hline \multicolumn{4}{|c|}{ Conoce persona que haya fallecido por COVID-19 } \\
\hline No & $135(49,09)$ & $392(33,31)$ & $<0,0001^{*}$ \\
\hline Sí & $140(50,91)$ & $785(66,69)$ & \\
\hline \multicolumn{4}{|c|}{ Cree usted que la COVID-19 es una enfermedad grave para su hijo/hija } \\
\hline No & $147(53,45)$ & $161(13,68)$ & $<0,0001^{*}$ \\
\hline Sí & $128(46,55)$ & $1016(86,32)$ & \\
\hline \multicolumn{4}{|c|}{ Ha pensado retirar a sus hijos temporalmente del colegio } \\
\hline No & $206(74,91)$ & $748(63,55)$ & $<0,0001^{*}$ \\
\hline Sí & $69(25,09)$ & $429(36,45)$ & \\
\hline \multicolumn{4}{|c|}{ Usted cree que la cuarentena ha traído problemas físicos a sus hijos } \\
\hline No & $153(55,64)$ & $795(67,54)$ & $<0,0001^{*}$ \\
\hline Sí & $122(44,36)$ & $382(32,46)$ & \\
\hline \multicolumn{4}{|c|}{ Si su respuesta fue SÍ, por favor elija al menos una de las siguientes opciones } \\
\hline No & $156(56,73)$ & $801(68,05)$ & $<0,0001^{\dagger}$ \\
\hline Sedentarismo & $7(2,55)$ & $29(2,46)$ & \\
\hline Obesidad & $39(14,18)$ & $178(15,12)$ & \\
\hline Retraso en el desarrollo & $67(24,36)$ & $148(12,57)$ & \\
\hline Accidentes en casa & $6(2,18)$ & $19(1,61)$ & \\
\hline Otros & $0(0,0)$ & $2(0,17)$ & \\
\hline \multicolumn{4}{|c|}{ Usted cree que la cuarentena ha traído problemas psicológicos a sus hijos } \\
\hline No & $98(35,64)$ & $678(57,60)$ & $<0,0001^{\ddagger}$ \\
\hline Sí & $177(64,36)$ & $499(42,40)$ & \\
\hline \multicolumn{4}{|c|}{ Si su respuesta fue SÍ, por favor elija al menos una de las siguientes opciones } \\
\hline No & $98(35,64)$ & $678(57,60)$ & $<0,0001^{\dagger}$ \\
\hline Ansiedad & $109(39,64)$ & $299(25,40)$ & \\
\hline Depresión & $36(13,09)$ & $96(8,16)$ & \\
\hline Otros & $32(11,64)$ & $104(8,84)$ & \\
\hline \multicolumn{4}{|c|}{$\begin{array}{l}\text { La respuesta de su hijo a la pregunta de si desea volver al colegio (PRESENCIAL) } \\
\text { (aplica para niños mayores de } 5 \text { años) }\end{array}$} \\
\hline No & $24(10,67)$ & $290(27,75)$ & $<0,0001^{\ddagger}$ \\
\hline Sí & $201(89,33)$ & $755(72,25)$ & \\
\hline
\end{tabular}

: Valor $\mathrm{p}$ determinado mediante prueba $x^{2}$.

¥: Valor p determinado mediante prueba U de Mann Whitney.

: Valor p determinado mediante prueba exacta de Fisher 
Al analizar las variables ajustadas por edad del padre, que fueron significativas para no aceptar el retorno a clases presenciales, los padres con hijo mayor de 10 años, (RP: 2,64; IC 95\%: 1,48-4,72; $p<0,001$ ), convivir con personas mayores de 70 años $(2,20$ : IC $95 \%$ : 1,46$3,29 ; p<0,0001)$ y conocer alguna persona que hubiera fallecido por COVID-19 también se encontraron asociadas (RP: 1,86; IC 95\%:1,42-2,44; $p<0,0001$ ). Al preguntarle a los padres si creían que la cuarentena trajo problemas físicos a sus hijos, se encontró significancia estadística en el retraso en el desarrollo (RP: 1,43; IC 95\%:1,31-1,61; $p<0,0001)$ y en los problemas psicológicos; se describieron la ansiedad (RP: 1.40; IC 95\%:1.29-1,54; $p<0,0001$ ), depresión (RP: 1,37; IC $95 \%$ : $1,24-1,58 ; p<0,0001)$ y otros problemas como trastorno de sueño, alimentación, agresividad, irritabilidad (RP: 1,46; IC 95\%: 1,29-1,72; $p<0,001$ ). Otras variables que se encontraban en la encuesta que estuvieron asociadas a la decisión de no retorno a clases presenciales se presentan en la Tabla 3.

Tabla 3. Variables asociadas a la decisión de no retorno a clases presenciales.

\begin{tabular}{|c|c|c|c|c|c|c|}
\hline \multirow{2}{*}{$\begin{array}{c}\text { Características } \\
\text { RP }\end{array}$} & \multicolumn{3}{|c|}{ Modelo crudo } & \multicolumn{3}{|c|}{ Modelo ajustado* } \\
\hline & $\begin{array}{c}\text { IC } \\
95 \%\end{array}$ & Valor $\mathbf{p}$ & $\mathbf{R P}$ & $\begin{array}{c}\text { IC } \\
95 \%\end{array}$ & Valor $\mathbf{p}$ & \\
\hline \multicolumn{7}{|l|}{ Padre o madre presenta alguna comorbilidad } \\
\hline Ninguna & Ref. & & & Ref. & & \\
\hline Hipertensión + infarto-angina & 3,24 & $2,07-5,08$ & $<0,0001$ & 2,99 & $1,89-4,75$ & $<0,0001$ \\
\hline Diabetes & 3,41 & $1,54-7,54$ & 0,002 & 3,24 & $1,46-7,21$ & 0,004 \\
\hline Obesidad & 2,88 & $1,67-4,95$ & $<0,0001$ & 2,87 & $1,66-4,94$ & $<0,0001$ \\
\hline Endocrinas & 2,04 & $0,90-4,63$ & 0,085 & 2,00 & $0,88-4,52$ & 0,096 \\
\hline Respiratorias & 2,09 & $1,22-6,86$ & 0,015 & 2,96 & $1,25-7,02$ & 0,013 \\
\hline Otras & 2,21 & $0,92-5,31$ & 0,074 & 2,18 & $0,91-5,24$ & 0,079 \\
\hline \multicolumn{7}{|c|}{ Convive con alguien que presente alguna comorbilidad } \\
\hline Ninguna & Ref. & & & Ref. & & \\
\hline Hipertensión + infarto-angina & 2,65 & $1,78-3,95$ & $<0,0001$ & 2,58 & $1,73-3,85$ & $<0,0001$ \\
\hline Diabetes & 2,47 & $1,38-4,43$ & 0,002 & 2,50 & $1,39-4,49$ & 0,002 \\
\hline Obesidad & 3,77 & $1,87-7,61$ & $<0,0001$ & 3,71 & $1,84-7,51$ & $<0,001$ \\
\hline Endocrinas & 2,21 & $1,25-3,91$ & 0,006 & 2,28 & $1,29-4,03$ & 0,005 \\
\hline Respiratorias & 4,85 & $1,14-20,56$ & 0,032 & 4,85 & $1,14-20,6$ & 0,032 \\
\hline Otras & 2,68 & $0,93-7,70$ & 0,066 & 2,80 & $0,97-8,06$ & 0,056 \\
\hline \multicolumn{7}{|c|}{$\begin{array}{l}\text { Cree usted que la COVID-19 es una enfermedad grave para su } \\
\text { hijo/hija }\end{array}$} \\
\hline No & Ref. & & & Ref. & & \\
\hline Sí & 7,24 & $5,42-9,68$ & $<0,0001$ & 7,92 & $5,88-10,6$ & $<0,0001$ \\
\hline \multicolumn{7}{|c|}{ Ha pensado retirar a sus hijos temporalmente del colegio } \\
\hline No & Ref. & & & Ref. & & \\
\hline Sí & 1,71 & $1,27-2,30$ & $<0,0001$ & 1,91 & $1,41-2,6$ & $<0,0001$ \\
\hline \multicolumn{7}{|c|}{$\begin{array}{l}\text { Usted estará de acuerdo en que se reanudaran las clases } \\
\text { presenciales }\end{array}$} \\
\hline No & Ref. & & & Ref. & & \\
\hline Sí & 0,031 & $0,02-0,04$ & $<0,0001$ & 0,027 & $0,17-0,04$ & $<0,0001$ \\
\hline \multicolumn{7}{|c|}{ Usted cree que arriesga la vida de su hijo si lo envía al colegio } \\
\hline No & Ref. & & & Ref. & & \\
\hline Sí & 17,11 & $11,85-24,70$ & $<0,0001$ & 19,25 & $13,1-28,2$ & $<0,0001$ \\
\hline \multicolumn{7}{|c|}{ Usted cree que su hijo desea volver a clases presenciales } \\
\hline No & Ref. & & & Ref. & & \\
\hline Sí & 0,17 & $0,10-0,29$ & $<0,0001$ & 0,16 & $0,09-0,27$ & $<0,0001$ \\
\hline \multicolumn{7}{|c|}{$\begin{array}{l}\text { La respuesta de su hijo a la pregunta de si desea volver al } \\
\text { colegio (presencial) (aplica para niños mayores de } 5 \text { años) }\end{array}$} \\
\hline No & Ref. & & & Ref. & & \\
\hline Sí & 0,31 & $0,99-0,48$ & $<0,0001$ & 0,30 & $0,19-0,47$ & $<0,0001$ \\
\hline
\end{tabular}


Además, se evaluaron los problemas físicos y psicológicos por edad de los niños desde el punto de vista de los padres (autopercepción); no se encontraron diferencias entre los grupos con $\mathrm{p}=0,132$ y $\mathrm{p}=0,163$.

\section{Discusión}

Este trabajo muestra que un porcentaje muy alto de padres no desea que sus hijos regresen a clases presenciales, en todas las etapas infantiles, lo cual incluye desde niños que acuden a guarderías hasta jóvenes en la secundaria. Es claro que la existencia de comorbilidades en padres y personas que convivan con ellos, como son hipertensión, cardiopatía isquémica $\mathrm{u}$ obesidad, son factores asociados para no aceptar el retorno a las clases presenciales. Ya es conocido que estos y otros factores como diabetes, enfermedades respiratorias, cáncer y ser fumadores son factores asociados con severidad en infección por COVID-199. Además, vivir con personas mayores de 70 años, con o sin comorbilidad, es otro factor asociado para no aceptar el retorno a clase de forma presencial ${ }^{10}$.

Algunas variables evaluaron el miedo a enfermar, por ejemplo, tener conocimiento de alguien cercano que haya fallecido por la enfermedad, posibilidad que se incrementaba a medida que trascurría la pandemia, esto genera temor, no solo por los cuidadores, sino también por sus hijos; este hecho está relacionado con mantener el aislamiento de los niños. Esta situación ya ha sido descrita por otros autores, quienes reportaron como factor asociado a mantener el aislamiento los altos niveles de temor a enfermar, considerándolo además como un comportamiento de salud preventiva que es más evidente en la población con más alto nivel educativo, sexo femenino, edad avanzada y residencia en zona urbana ${ }^{11}$. Otros trabajos similares han encontrado que el porcentaje de mantener a los hijos en casa oscila alrededor del $50 \%$ de los casos. Los factores evaluados, asociados a mantenerse en casa, fueron los bajos ingresos, estar desempleados y tener un trabajo flexible; además, el miedo a la enfermedad, el síndrome inflamatorio multi-sistémico, la poca confianza en las escuelas y los desafíos de la educación en el hogar ${ }^{12}$. Este último, en una población de estratos sociales más dispersos, lo que claramente demuestra que el problema es global. Si bien, este trabajo no realiza una evaluación en todos los niveles económicos, la problemática podría ser global, pero por condiciones diferentes, como puede ser el factor económico en la población de menos nivel socioeconómico ${ }^{12}$.
A partir de los hallazgos anteriores sobre los motivos para no aceptar el retorno a los colegios, hay dos premisas que discutir. La primera está en relación con la gravedad de la enfermedad en niños. Si bien la información es aún escasa, hay algunos datos que podrían ayudar a resolver esta pregunta. Maltezou, et al. evaluaron 203 niños con infección por COVID-19 con edades entre los 6 días y 18 años; de estos, $111(54,7 \%)$ fueron asintomáticos, 92 presentaron síntomas, y de estos 92 , solo 24 (26\%) requirieron hospitalización. Los niños menores de un año fueron los más propensos a desarrollar enfermedad sintomática y representaban el $20 \%$ de todos los $\operatorname{casos}^{13}$. De acuerdo con datos de la literatura mundial, los niños representan entre el $1-5 \%$ de los casos diagnosticados de COVID-19. La presentación clínica es menos severa que en los adultos, $90 \%$ son asintomáticos y una minoría tiene síntomas de leves a moderados. Sin embargo, aproximadamente, un $7 \%$ puede desarrollar síntomas severos y, generalmente, son niños menores de un año o con alguna enfermedad de base ${ }^{14}$. Sin bien, esto sigue prevaleciendo, en la última ola de la pandemia hay una tendencia de compromiso en personas más jóvenes, condición que debe ser vigilada, incluyendo la población infantil ${ }^{15}$.

Cabe destacar que, de forma similar a los adultos, los niños con afecciones médicamente complejas, los obesos y con enfermedades crónicas tienen un mayor riesgo de contraer la infección por COVID-19 y un comportamiento clínico más complejo. Es probable que la principal preocupación sea el síndrome de respuesta inflamatoria sistémica en niños, que es una condición rara, con reportes al 6 de agosto de 550 casos y 10 muertes ${ }^{16}$, principalmente entre niños de 10 a 16 años. Si bien, es una complicación grave, hay opciones de manejo con resultados favorables ${ }^{17}$. Al momento de definir la reapertura de las escuelas, es clave tener en cuenta, como un sustrato importante, la información que responde a la primera premisa sobre la severidad de la morbimortalidad en niños asociada a COVID-19, que afortunadamente es baja $\mathrm{a}^{18}$.

La segunda premisa es el riesgo real de que los niños sean trasmisores de la enfermedad a las personas que convivan con ellos, con o sin factores de riesgo. En este sentido, es poco probable que ellos, principalmente los más pequeños, adopten medidas apropiadas para disminuir la probabilidad de contagio, como son el lavado de manos, evitar tocarse la cara, uso de mascarillas y el manejo de la higiene de la tos. Esto sumado a los espacios reducidos en los colegios y 
a las múltiples interacciones sociales, hace de los colegios espacios propicios para diseminar el virus, no solo entre los niños, sino también a profesores y trabajadores en general. Sin embargo, es importante evaluar esta hipótesis ${ }^{19}$. Datos recientes anotan que el comportamiento puede ser diferente según la edad de los niños. Si bien, un estudio antes mencionado (15) informa que la trasmisión de la infección de un miembro de la familia se pudo documentar en el $74,2 \%$ de los casos de niños con COVID-19 (adulto a niño), solo se pudo comprobar un caso de infección de un niño transmitido a un adulto. Esto aplica en todos los grupos, incluso en los menores de un año ${ }^{20}$. Otro trabajo anota que los niños menores de diez años tienen menos transmisibilidad en comparación con los niños por encima de esta edad ${ }^{21}$, condición que debe tenerse en cuenta al momento de tomar decisiones en salud pública e infantil; este dato coincide con los hallazgos del presente estudio, donde se encontró que los padres con niños mayores de diez años consideran esta edad como un factor asociado a la decisión de mantener la virtualidad.

Con las evidencias actuales, los niños se consideran menor fuente de trasmisión al compararse con los adultos, por lo tanto, usar como argumento para que no retornen al colegio el potencial riesgo para contagiar a padres con comorbilidades o a adultos mayores no tendría una clara justificación ${ }^{22}$, pero el riesgo no debe ser excluido en su totalidad. Por lo anterior, los niños no serían vectores importantes para COVID-19, a diferencia de otros virus respiratorios ${ }^{23}$; por esto, es una razón de poco peso al tomarla como parte de la decisión final de no aceptar un retorno a clase de forma presencial.

Como se anotó, hay una clara posición de rechazo de los padres para que sus hijos regresen a las aulas escolares. No obstante, hay recomendaciones de gobiernos y sociedades científicas para el retorno de forma presencial de los estudiantes. La Academia Americana de Pediatría (AAP) propone más atención y diseño de políticas en relación con el cierre de las clases presenciales; plantea sitios de aprendizaje alternativo, modelos educativos híbridos y vigilancia de salud pública en relación con el cuidado de los niños. Además, las escuelas son fundamentales para el desarrollo y bienestar de niños y adolescentes, no solo en el aspecto académico, sino también en habilidades sociales, emocionales y oportunidades para la actividad física $^{24}$. Sumado a esto, la escuela podría ser una opción de soporte nutricional, servicios de terapia de lenguaje, salud mental, entre otras opciones; esto podría tener mayor impacto en países de bajos ingresos. Ya otras sociedades como la Asociación de Pediatría de Sur África (SAPA), basada en la literatura actual, promueve el regreso presencial a las escuelas, pues los niños de bajos recursos tienen limitación de acceso en línea y, además, pierden el beneficio de alimentación escolar ${ }^{25}$. Si bien estos puntos son integrales, el cognitivo podría ser el más relevante. A la evidencia actual, el impacto cognitivo se presenta solo con el cierre de ocho semanas de colegio presencial (ejemplo: Holanda), condicion que puede ser mas severa, hasta un $60 \%$ de compromiso cognitivo en hogares con menor educación y con mayor tiempo de estudio virtual, lo que refleja una brecha educativa amplia generada por la pandemia. Los trabajos recientes encuentran que los estudiantes hicieron poco esfuerzo por aprender, situación marcada en países con infraestructuras educativas débiles o con cierre de escuelas más prolongados ${ }^{26}$.

Asimismo, para varios autores, las medidas de mantener el cierre de las escuelas no son un factor contribuyente para el control de la pandemia, incluso, ya con aproximaciones, esta medida solo podría prevenir entre 2 y $4 \%$ de las muertes ${ }^{27}$ y puede ser más perjudicial que beneficiosa; por lo anterior, debe ser considerada como última estrategia en las medidas de prevención de propagación. Además, existen medidas ya establecidas para el regreso (alternancia), donde la más importante está en relación con la prevención y manejo de los casos sospechosos y confirmados ${ }^{28}$. A pesar de la anterior, después de 12 meses de cierre y de apertura de otros espacios sociales, las escuelas permanecen cerradas. Solo algunos han buscado la alternancia. El cierre prolongado priva a los niños de experiencias necesarias para su pleno desarrollo, especialmente en edades donde las necesitan. También, tiene un impacto negativo en la capacidad de pensamiento crítico y en las habilidades sociales, sin mencionar problemas específicos según edades como puede ser en adolescentes el embarazo precoz, reclutamiento por grupos armados, entre otros. Algunas condiciones como el abuso físico y sexual, también, puede aparecer durante esta prolongación del cierre de colegios $^{23}$. Otros aspectos para evaluar son los desenlaces negativos en lo biopsicosocial, como consecuencia del confinamiento prolongado. En lo psicológico, se sabe por datos preliminares que en niños y adolescentes la pandemia y el confinamiento tiene un mayor impacto emocional y social en comparación con los adultos. Algunas alteraciones del comportamiento como irritabilidad, falta de atención y alteración del apego fueron detectadas en todos los grupos etarios, al igual que otros síntomas como inseguridad, temor, alteraciones del sueño, apetito (hiperfagia o hiporexia), agitación y ansiedad, relacionados con la separación ${ }^{29}$. 
Muchos de estos síntomas son marcadores de depresión en la población infantil ${ }^{30}$. Si bien, la edad podría ser un factor que hace a los niños mayores más vulnerables a la depresión ${ }^{31}$, esto no fue encontrado en el presente reporte. El otro síntoma expresado fue la ansiedad, el cual es homogéneo en todos los grupos de edad. Trabajos recientes reportan presencia de ansiedad entre el 19,4\% y $21,8 \%$ de los niños, esto además muestra que los niños en edad escolar y las niñas son más susceptibles a presentar ansiedad ${ }^{32}$. Es importante anotar que los padres que desean el retorno a clase son los que más expresan estos síntomas en sus hijos. Las alteraciones físicas también fueron reportadas, una de ellas fue la obesidad, posiblemente relacionada con el aumento de la exposición a pantallas, cambios en los hábitos alimentarios y disminución en la actividad física ${ }^{33}$. Esta última, también ha sido postulada como importante en la salud mental durante periodos de confinamiento ${ }^{31}$.

Otros aspectos para evaluar son las alteraciones de neurodesarrollo y conducta. Esto ha sido más relevante entre el grupo de padres que desean las clases presenciales. Por lo anterior, se deben valorar los impactos agudos y a largo plazo, porque podrían estar en relación con problemas de reintegración escolar, rendimiento académico y aparición de problemas de conducta ${ }^{15}$. Por otro lado, uno de cada seis niños tiene problemas en el desarrollo neurológico, conductual o emocional, con mayores consecuencias negativas, e incluso regresión de sus logros. Atención especial merecen los pacientes con autismo, hiperactividad y déficit de atención y desórdenes obsesivos convulsivos, quienes tienen mayor dificultad para adaptarse a estos nuevos cambios ${ }^{29,34}$. Otros problemas como desigualdad en la educación y en la salud aparecerán con mayor impacto en la población vulnerable, debido a la pobreza en sí, a la presencia de pacientes con enfermedades crónicas, discapacidad, necesidades educativas especiales y a la limitación de acceso del aprendizaje a distancia ${ }^{29,31,34}$.

\section{Fortalezas y limitaciones}

La principal fortaleza de este estudio es ser el primero que se lleva a cabo en el territorio colombiano. Una de las limitaciones es que no se preguntó el nivel socioeconómico ni la seguridad social de los participantes; sin embargo, es seguro que sean de niveles medios y altos por el tipo de padres que acude a los consultorios involucrados. Se considera, con mucha cautela, que la población encuestada de mayor nivel socioeconómico tiene un sentido de autocuidado mayor y que la percepción de niveles bajos puede ser diferente, como ha sido reportada en otros estudios ${ }^{9}$, $\mathrm{y}$, probablemente, en nuestra población esta variable juegue un papel importante para aceptar o no el retorno a las clases presenciales.

Otra limitación en este estudio es que no se determinaron las comorbilidades en los niños, factor para tener en cuenta en la toma de una decisión para el retorno al colegio. Por otro lado, en relación con los efectos físicos y sociales, estos fueron determinados por la percepción de los padres, y no fueron evaluados por un profesional. Creemos que los padres pueden observar potenciales cambios de comportamiento y alteraciones físicas como las evaluadas en la encuesta. Es importante evaluar la percepción de todos los involucrados en la apertura, como es la posición de los profesores sobre este tópico, situación no menos relevante. Asimismo, es importante la condición variable de la pandemia, lo que a su vez puede ser un factor determinante en algunas percepciones sobre volver a actividades pre-pandemia.

\section{Conclusiones}

Existe una clara tendencia a mantener la virtualidad como opción educativa en Colombia, al menos en la población evaluada: estrato medio y alto; los factores asociados a esta decisión están basados en percepciones de miedo por la pandemia, no confirmados desde lo científico. Por otro lado, la persistencia de clases virtuales no está libre de efectos adversos, lo cuales se pueden profundizar si los niños continúan en una escolaridad virtual. Las políticas serán dinámicas, pero evaluar de forma integral las decisiones será un reto inmediato. Finalmente, hay que buscar opciones de regreso al colegio teniendo a favor todos los argumentos antes enunciados y propender porque las políticas gubernamentales aporten los recursos suficientes para que los espacios físicos ofrezcan las condiciones de bioseguridad, sanidad física y mental, seguridad alimentaria y de conectividad necesarias para este retorno gradual e inteligente, adaptándose a las circunstancias propias de cada región y a la evolución impredecible de esta pandemia. Los gobiernos deberían pensar que estos hallazgos y decisiones de padres tienen implicaciones sobre la discriminación y fragmentación de la sociedad. Además, se debe mejorar la virtualidad de los más desfavorecidos, diseñar y promover presencialidad de bajo riesgo, en el momento de la decisión de retornar de forma presencial a los colegios. 


\section{Agradecimientos}

A los pediatras: Idali Amado, María Isabel Camacho, Camilo Ortiz, Camilo Luna, Ángela Puerta, Ximena Córdoba, Rafael Bolaño y Bertha Inés Agudelo quienes participaron en la difusión de la encuesta en diferentes ciudades del país.

\section{Consideraciones éticas}

El estudio se realizó de acuerdo con la Declaración de Helsinki. La elaboración y ejecución de esta investigación cumplió a cabalidad los principios éticos fundamentales de autonomía, justicia, beneficencia y no maleficencia. Se dio cumplimiento a los planteamientos éticos consignados en el código de ética médica (Ley 23 de 1981) y en la Resolución 8430 de 1993 del Ministerio de Salud de Colombia, que establecen las normas para la investigación en salud en la que participan seres humanos. De acuerdo con esta resolución, este se considera un estudio sin riesgo, por lo referido en el artículo 11, numeral a.

Por lo anteriormente mencionado, y dadas las condiciones de emergencia sanitaria, durante la planeación del estudio, necesario para decisiones de salud pública, se decide la implementación de la encuesta sin presentación al comité de ética, dadas, en su momento, las prioridades de investigación de mayor riesgo ético, por ejemplo, vacunación, pruebas diagnósticas, medicaciones, entre otras. Debido a la situación actual de la crisis sanitaria, se permite el desarrollo de trabajos basados en cambios y flexibilidad de lo ético en el momento de crisis sanitarias $^{35,36}$. Por lo anterior, no fue sometido al comité institucional de ética, dada la necesidad de información que permita visualizar soluciones rápidas a un serio problema en la población infantil.

\section{Conflicto de Interés}

Los autores declaran que no existe ningún conflicto de interés.

\section{Referencias}

1. Fisher D, Teo YY, Nabarro D. Assessing national performance in response to COVID-19 -Lancet. 2020; 396(10252): 653-655. doi: 10.1016/S01406736(20)31601-9

2. Rajgor DD, Lee MH, Archuleta S, Bagdasarian N, Quek SC. The many estimates of the COVID-19 case fatality rate. The Lancet Infect Diseases. 2020; 20(7): 776-777. doi: doi.org/10.1016/S14733099(20)30244-9
3. Kaur S, Bherwani H, Gulia S, Vijay R, Kumar Rakesh. Understanding COVID-19 transmission, health impacts and mitigation: timely social distancing is the key. Environ Dev Sustain. 2020; 18: 1-17. doi: 10.1007/s10668-020-00884-X

4. Hiscott J, Alexandridi M, Muscolini M, Tassone E, Palermo, Soultsioti M, Zevini A. The global impact of the coronavirus pandemic. Cytokine Growth Factor Rev. 2020; 53: 1-9. doi: 10.1016/j. cytogfr.2020.05.010

5. Bonell C, Melendez-Torres GJ, Viner RM, Brooke Rogers M, Whitworth M, Rutter $\mathrm{H}$, et al. An evidence-based theory of change for reducing SARS-CoV-2 transmission in reopened schools. Health Place. 2020; 64:102398. doi: 10.1016/j. healthplace.2020.102398

6. Jackson C, Mangtani P, Hawker J, Olowokure B, Vynnycky E. The effects of school closures on influenza outbreaks and pandemics: systematic review of simulation studies. PLoS One. 2014; 9(5): e97297. doi: 10.1371/journal.pone.0097297

7. Lee J. Mental health effects of school closures during COVID-19. Lancet Child Adolesc Health. 2020; 4(6): 421. doi: 10.1016/S2352-4642(20)30109-7

8. Jeffs E, Lucas N, Walls T. CoVID-19: Parent and caregiver concerns about reopening New Zealand schools. J Paediatr Child Health. 2021; 57: 403-408. doi: 10.1111/jpc.15234

9. Jordan RE, Adab P, Cheng KK. Covid-19: risk factors for severe disease and death. BMJ. 2020; 26; 368: m1198. doi: https://doi.org/10.1136/bmj. $\mathrm{m} 1198$

10. Setiati S, Harimurti K, Safitri ED, Ranakusuma RW, Saldi SR, Azwae MK, et al. Risk factors and laboratory test results associated with severe illness and mortality in COVID-19 patients: A systematic review. Acta Med Indones. 2020; 52(3): 227-245.

11. Hossain MA, Jahid MIK, Hossain KMA, Walton LM, Uddin Z, Haque MO, et al. Knowledge, attitudes, and fear of COVID-19 during the rapid rise period in Bangladesh. PLoS ONE. 2020; 15(9): e0239646. doi: doi.org/10.1371/journal.pone.0239646

12. Kroshus E, Hawrilenko M, Tandon P, Christakis D. Plans of US parents regarding school attendance for their children in the fall of 2020. JAMA Pediatr. 2020; 174(11): 1-10. doi 10.1001/ jamapediatrics.2020.3864

13. Maltezou HC, Magaziotou I, Dedoukou X, Eleftheeriou E, Raftopoulos V, Michos A, et al. Children and Adolescents with SARS-CoV-2 Infection: Epidemiology, Clinical Course and Viral Loads. Pediatr Infect Dis J. 2020; 39(12): 388-392. doi: 10.1097/INF.0000000000002899 
14. Tezer H, Demirdağ TB. Novel coronavirus disease (COVID-19) in children. Turk J Med Sci. 2020;50 (S1-1): 592-603. doi: 10.3906/sag-2004-174

15. Altmann DM. Children and the return to school: how much should we worry about covid-19 and long covid? BMJ. 2021; 15; 372: n701. doi: 10.1136/ bmj.n701

16. Betz CL. COVID-19 and school return: The need and necessity. J Pediatr Nurs. 2020; 54: A7-A9. doi: 10.1016/j.pedn.2020.07.015

17. Belhadjer Z, Meot M, Bajolle F, Khraiche D, Legendre A, Abakka S, et al. Acute heart failure in multisystem inflammatory syndrome in children (MIS-C) in the context of global SARS-CoV-2 pandemic. Circulation. 2020; 142(5): 429-436. doi: 10.1161/CIRCULATIONAHA.120.048360

18. Dong Y, Mo X, Hu Y, Qi X, Jiang F, Jiang Z, et al. Epidemiological characteristics of 2143 pediatric patients with 2019 Coronavirus disease in China. Pediatrics. 2020; 145: e20200702. doi: 10.1542/ peds.2020-0702

19. Hoffman J, Miller E. Addressing the consequences of school closure due to COVID-19 on children's physical and mental well-being. World Med Health Policy. 2020; doi: 10.1002/wmh3.365

20. Theurel-Martin DE, Gomez A, Saldarriaga D, Villegas-Lamus KC, Alvarado-Socarras JL. SARSCoV2 como causa de sepsis neonatal tardía, reporte de un caso. Salud UIS. 2020; 53(4): 456-460. doi: https://doi.org/10.18273/revsal.v52n4-2020013

21. Cho EY, Choi EH, Kim JH. Interpreting transmissibility of COVID-19 in children. Emerg Infect Dis. 2020; 26(12): 3106-3107. doi: 10.3201/ eid2612.203452

22. Zhu Y, Bloxham CJ, Hulme KD, Sinclair JE, Marcus Tong ZW, Steele LE, et al. Children are unlikely to have been the primary source of household SARS-CoV-2 infections. SSRN J. 2020. doi: 10.11 01/2020.03.26.20044826

23. Buonsenso D, Roland D, De Rose C, VásquezHoyos P, Ramly B, Chakakala-Chaziya JN et al. Schools closures during the covid-19 pandemic: A catastrophic global situation. Pediatr Infect Dis J. 2021; 40(4): 146-150. doi: 10.1097/ INF.0000000000003052

24. American Academy of Pediatrics. COVID-19 Planning Considerations: Guidance for School Reentry; 2020.

25. Hendricks C, Green R. COVID-19 in children: Should we be worried? South African Med J. 2020; 110(9): 864. doi: 10.7196/SAMJ.2020. v110i9.15023
26. Engzell P, Frey A, Verhagen MD. Learning loss due to school closures during the COVID-19 pandemic. Proc Natl Acad Sci USA. 2021; 118(17): e2022376118. doi: 10.1073/pnas.2022376118

27. Viner R, Russell SJ, Croker H, Packer J, Ward J, Stansfield C, et al. School closure and management practices during coronavirus outbreaks including COVID-19: a rapid systematic review. Lancet Child Adolesc Health. 2020; 4(5): 397-404. doi: https:// doi.org/10.1016/S2352-4642(20)30095-X

28. Lo Moro G, Sinigaglia T, Bert F, Savatteri A, Gualano MR, Siliquini R. Reopening Schools during the COVID-19 Pandemic: overview and rapid systematic review of guidelines and recommendations on preventive measures and the management of cases. Int $\mathrm{J}$ Environ Res Public Health. 2020; 17(23): 8839. doi: 10.3390/ ijerph 17238839

29. Singh S, Roy D, Sinha K, Parveen S, Sharma G, Joshi G. Impact of COVID-19 and lockdown on mental health of children and adolescents: A narrative review with recommendations. Psychiatry Res. 2020; 293: 113429. doi: 10.1016/j. psychres.2020.113429

30. Du L, Chen YM, Jin X, Yuan W, Wang JS. Critical appraisal of clinical practice guidelines for depression in children and adolescents: A protocol for systematic review. Medicine (Baltimore). 2020; 99(38): 22384. doi: 10.1097/ MD.0000000000022384

31. Marques de Miranda D, da Silva Athanasio B, Sena Oliveira AC, Simoes-E-Silva AC. How is COVID-19 pandemic impacting mental health of children and adolescents? Int J Disaster Risk Reduct. 2020; 51: 101845. doi: 10.1016/j.ijdrr.2020.101845

32. García de Ávila MA, Hamamoto Filho PT, Da silva Jacob FL, Souza Alcantara LR, Berghammer M, Jenholt Nolbris M, et al. Children's anxiety and factors related to the COVID-19 Pandemic: An exploratory study using the children's anxiety questionnaire and the numerical rating scale. Int J Environ Res Public Health 2020; 17(16): 5757. doi: 10.3390/ijerph 17165757

33. López Bueno R, López Sanchez G, Casajus JA, Calatayud J, Gil-Salmerón A, Grabovac I, et al. Health-related behaviors among school-aged children and adolescents during the Spanish Covid-19 Confinement. Front Pediatr. 2020; 8: 573. doi: 10.3389/fped.2020.00573

34. Nonweiler J, Rattray F, Baulcomb J, Happé F, Absoud M. Prevalence and associated factors of emotional and behavioural difficulties during COVID-19 
pandemic in children with neurodevelopmental disorders. Children 2020; 7(9) 128. doi: 10.3390/ children7090128

35. Reyes M. Research in the Time of COVID-19: Challenges of research ethics committees. J ASEAN
Fed Endoc Societies. 2020; 35(1): 29-32. doi: 10.15605/jafes.035.01.07

36. Guidance for Managing Ethical Issuesisêpin Infectious Disease Outbreaks. World Health Organization; 2016.

\section{NOTA EDITORIAL}

Salud UIS recibió y evaluó el manuscrito original y sus versiones modificadas posteriores a las evaluaciones realizadas. Desde el inicio se detectó que el estudio carecía de un aval por un comité de ética. Por tal razón, solicitó a los autores detalles pormenorizados de este (la mayoría plasmados en la versión aquí impresa), y dado que el tema es de vital importancia para la toma de decisiones se decidió publicarlo. Se puede concluir que el estudio es de riesgo mínimo, y lo que se hizo no traía mayores riesgos para los padres de familia o los menores de edad. Sin duda, el protocolo de éste hubiese sido aprobado por un comité de ética. Dado lo sucedido se invitó a los autores en el futuro no incurrir en este error y se publica en Salud UIS por la importancia coyuntural durante la pandemia.

Álvaro J Idrovo Editor Salud UIS 